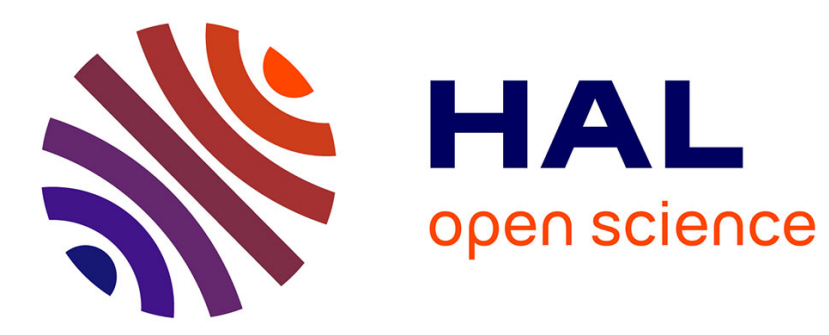

\title{
Kinetostatic Modeling of a Cable-Driven Parallel Robot Using a Tilt-Roll Wrist
}

\author{
Saman Lessanibahri, Philippe Cardou, Stéphane Caro
}

\section{To cite this version:}

Saman Lessanibahri, Philippe Cardou, Stéphane Caro. Kinetostatic Modeling of a Cable-Driven Parallel Robot Using a Tilt-Roll Wrist. In: Pott A., Bruckmann T. (eds) Cable-Driven Parallel Robots. CableCon 2019. Mechanisms and Machine Science, vol 74. Springer, Cham, pp.109-120, 2019, 10.1007/978-3-030-20751-9_10 . hal-02404449

\section{HAL Id: hal-02404449 \\ https://hal.science/hal-02404449}

Submitted on 11 Dec 2019

HAL is a multi-disciplinary open access archive for the deposit and dissemination of scientific research documents, whether they are published or not. The documents may come from teaching and research institutions in France or abroad, or from public or private research centers.
L'archive ouverte pluridisciplinaire HAL, est destinée au dépôt et à la diffusion de documents scientifiques de niveau recherche, publiés ou non, émanant des établissements d'enseignement et de recherche français ou étrangers, des laboratoires publics ou privés. 


\title{
Kinetostatic Modeling of a Cable-Driven Parallel Robot using a Tilt-Roll Wrist
}

\author{
Saman Lessanibahri ${ }^{1,2}$, Philippe Cardou ${ }^{3}$, and Stéphane Caro $^{2,4}$ \\ 1 École Centrale de Nantes, Nantes, 44321 France, \\ 2 Laboratoire des Sciences du Numérique de Nantes (LS2N), UMR CNRS 6004, \\ Nantes, 44300, France, \\ 3 Département de génie mécanique, Université Laval, Québec, QC, Canada, \\ 4 Centre National de la Recherche Scientifique (CNRS), Nantes, 44321, France, \\ Emails: Saman.Lessanibahri@ls2n.fr \\ Philippe.Cardou@gmc.ulaval.ca, Stephane.Caro@ls2n.fr
}

\begin{abstract}
This paper introduces the concept of a new Cable-Driven Parallel Robot (CDPR) applicable for tasks requiring large rotation and translation workspaces, e.g., camera orienting devices, visual surveillance over vast areas and tomography scanners. The manipulator consists of a fixed frame and a tilt-roll wrist attached to a Moving-Platform (MP). The MP is an under-constrained and articulated mechanism connected to the fixed frame with six cables. The power is transmitted directly from the motors fixed on the frames to the tilt-roll wrist through cable loops. While most of the CDPRs only provide a limited range of rotation of the MP, the proposed manipulator is applicable for a relatively large and singularity-free rotation workspace thanks to its articulated MP.
\end{abstract}

Keywords: novel mechanism, cable loop, tilt-roll wrist, large orientation workspace

\section{Introduction}

There exists a wide variety of applications requiring large rotation workspace, e.g., search and rescue, motion simulation and entertainment. Cable-Driven Parallel Robots (CDPRs) have drawn their interest of industry thanks to their fundamental advantages and capabilities, such as high payload-to-weight ratios, large translational workspaces and high-speed motions. They are parallel robots with cables instead of rigid links and they can provide large translational workspaces. They generally cannot cover large rotation workspaces due to collisions between their moving parts. This paper introduces a hybrid robot with a large translational and rotation workspace consisting in a CDPR connected to a tilt-roll wrist. The manipulator combines the advantages of CDPRs, i.e., a large translational workspace, with those of tilt-roll wrists, namely, large amplitudes of rotation one. Solution to overcome the limited rotation workspace of CDPRs could consist in mounting a standard tilt-roll wrist on the Moving-Platform (MP) of a CDPR, but articulation of the tilt-roll wrist could be a challenge. Adding 
motors to the MP for actuating the wrist increases the weight of the MP and reduces the dynamic performance of the manipulator. Therefore, a pair of biactuated cable circuits, namely, cable loops are employed in the design of the CDPR in order to transmit the power from the motors fixed on the frame to the tilt-roll wrist. In general, the workspace of parallel robots can expand by connecting other parallel or serial mechanisms to them. This usually complicates the design and reduces the dynamic performance of the manipulator unless, the power is transmitted directly to the end-effector without locating motors on the MP. By employing remote actuation, we avoid mass and entanglement of a long power line tethering the MP to the ground. Moreover, because of the mentioned reasons, remote actuation minimizes the size of the MP. This leads to a better dynamic performance due to the lower inertia of the MP. Hence, coupling two parallel mechanisms is proposed in order to exploit their combined workspaces through cable loops. Many applications require pointing the end-effector over a wide range of directions such as, camera surveillance, laser scanning, tomographic scanner, etc.

Cable loops have been addressed in several papers [7], [13] and it was employed in [10] for actuation of a hoist mechanism embedded in a MP. In the latter the large rotational amplitudes provided by a cable loop was used to actuate a one Degree of Freedom (DoF) mechanism, while here we employ two cable loops to actuate a tilt-roll wrist having a large rotational workspace. H. Khakpour et al. in [4],[5] and [6] introduced differentially driven cable parallel robots using cable differentials. They proved that, by replacing single-actuated cables with differential cables the static and wrench feasible workspaces of CDPRs can be extended.

The study of the concept of the hybrid CDPR providing a large translational and rotational workspace is organized as follows. Section 2 introduces the architecture of the under-constrained manipulator with eight DoF. Section 3 describes the kinetostatic model of the tilt-roll wrist. The kinetostatic model of the overall manipulator is presented in Sec. 4. Section 5 investigates the static workspace of the CDPR and the last section concludes the paper.

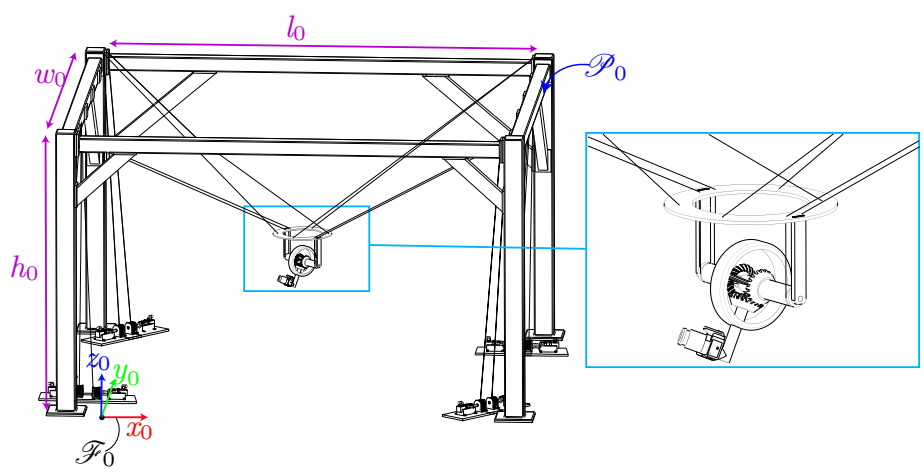

Fig. 1: Schematic of the CDPR using a Tilt-Roll Wrist 


\section{Manipulator Architecture}

Here, we present the architecture of the manipulator, which is a hybrid robot consisting of a base frame and a tilt-roll wrist connected to the MP of a CDPR. The MP is suspended by six cables, i.e., two bi-actuated cable circuits, also named cable loops, and four uni-actuated cables as illustrated in Fig. 1.

A cable loop forms a circuit by connecting two actuators while passing through two anchor points on the MP and coiling about a gear on the tilt-roll wrist. This arrangement of cables is used for two distinct purposes. The former is to locate the MP within its workspcae and the latter is to actuate the tilt-roll wrist connected in series to the MP. In other words, two motions can be induced by the cable loop depending on the relative rotation of its two actuators. The first one is the displacement of the MP for identical inputs to the two motors. The second motion is the rotation of gears $\mathscr{P}_{3}$ and $\mathscr{P}_{4}$ about their axes $\left(z_{3}\right.$ and $z_{4}$ ), shown in Fig. 2, when the two actuators rotate in opposite directions. This leads to an unlimited rotation of $\mathscr{P}_{3}$ and $\mathscr{P}_{4}$ about their respective rotation axes. Figure 3 describes the main geometrical parameters of the MP as well as the tilt-roll wrist. A cable loop connected to two motors and drums is highlighted in Fig. 3. Cable loop consists of two segments each connecting the drums to their anchor points on the MP with independent tensions and velocities.

The MP frame $\mathscr{F}_{1}$ is located on the top of the MP at point $P .{ }^{0} \mathbf{p}$ is the vector pointing from the origin of $\mathscr{F}_{0}$ to the origin of $\mathscr{F}_{1} .{ }^{0} \mathbf{R}_{1}$ is the rotation matrix from frame $\mathscr{F}_{0}$ to frame $\mathscr{F}_{1}$ and it is expressed as three sequence of rotations, i.e., ${ }^{0} \mathbf{R}_{1}=\mathbf{R}_{z}(\psi) \mathbf{R}_{y}(\chi) \mathbf{R}_{x}(\phi) . \alpha$ and $\beta$, shown in Fig. 4, characterize the two-DoF rotational motions of the tilt-roll wrist.

\section{Kinetostatic Model of the Tilt-Roll Wrist}

Kinetostatic model of the tilt-roll wrist is investigated in this section. The section-view of the MP and the wrist is shown in Fig. 2. Their main components are denoted as $\mathscr{P}_{i}, \quad i=1, \ldots, 5$. The MP is represented with $\mathscr{P}_{1}$, carrier of the wrist is denoted as $\mathscr{P}_{2}$, input gears connected to the cable loops $\mathscr{C}_{12}$ and $\mathscr{C}_{56}$ being $\mathscr{P}_{3}$ and $\mathscr{P}_{4}$, respectively. $\mathscr{P}_{5}$ is the end-effector.

Figure 4 shows the parametrization of the tilt-roll wrist in terms of angles. The rotation angles of $\mathscr{P}_{2}, \mathscr{P}_{3}$ and $\mathscr{P}_{4}$ about $z_{2}$-axis are denoted as $\alpha=\angle\left(z_{2}, x_{5}\right), \theta_{3}=\angle\left(y_{1}, x_{3}\right)$ and $\theta_{4}=\angle\left(y_{1}, x_{4}\right)$, respectively. The roll angle of the end-effector is defined as $\beta=\angle\left(z_{2}, x_{5}\right)$. The gear train ratio, $\mu$ is expressed as:

$$
\mu=\frac{r_{5}}{r_{3}}=\frac{N_{5}}{N_{3}}
$$

where $N_{3}$ and $N_{5}$ are the number of teeth of gears $\mathscr{P}_{3}$ and $\mathscr{P}_{5}$. The pitch radius of $i$ th gear is denoted as $r_{i}$ and $i=3,4,5$. It should be noted that $r_{3}=r_{4}$. The linear velocities at gear contact points $C_{35}$ and $C_{45}$ are expressed as follows:

$$
{ }^{1} \mathbf{v}_{c 35}=-\dot{\theta}_{3} r_{3}{ }^{1} \mathbf{y}_{2}
$$




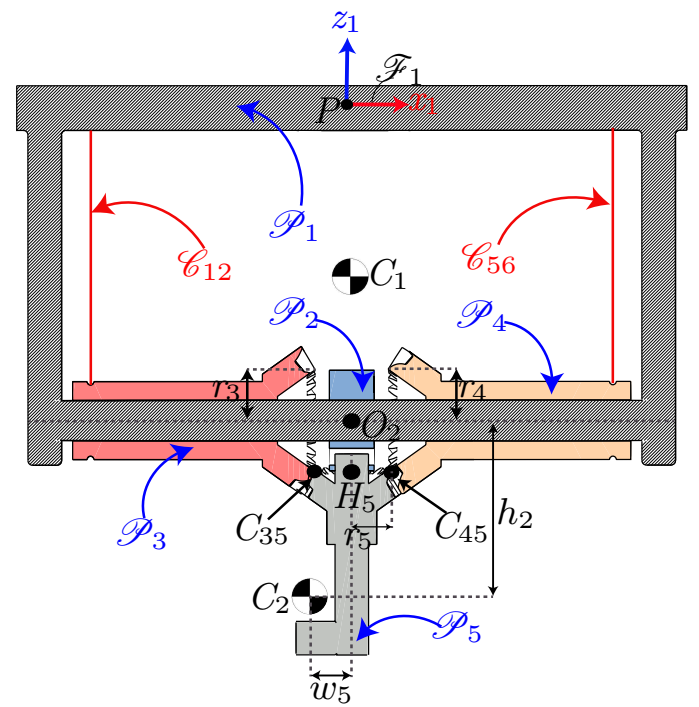

Fig. 2: Section-view of the moving-platform and tilt-roll wrist

$$
{ }^{1} \mathbf{v}_{c 34}=-\dot{\theta}_{4} r_{4}{ }^{1} \mathbf{y}_{2}
$$

with $\dot{\theta}_{3}$ and $\dot{\theta}_{4}$ being the angular velocities of $\mathscr{P}_{3}$ and $\mathscr{P}_{4}$, respectively.

$$
{ }^{1} \mathbf{v}_{H 5}=-r_{3} \dot{\alpha}^{1} \mathbf{y}_{2}
$$

${ }^{1} \mathbf{v}_{H 5}$ is the linear velocity of point $H_{5}$ which belongs to $\mathscr{P}_{5}$ expressed in $\mathscr{F}_{1}$. Furthermore, the angular velocity of the end-effector $\mathscr{P}_{5}$ with respect to $\mathscr{F}_{1}$ and expressed in $\mathscr{F}_{1}$ takes the form:

$$
{ }^{1} \omega_{5 / 1}=\dot{\alpha}^{1} \mathbf{z}_{2}-\dot{\beta}^{1} \mathbf{x}_{2}
$$

Knowing the linear velocity ${ }^{1} \mathbf{v}_{H 5}$ of point $H_{5}$ in $\mathscr{F}_{1}$, the linear velocities ${ }^{1} \mathbf{v}_{c 35}$ and ${ }^{1} \mathbf{v}_{c 45}$ of points $\mathbf{c}_{35}$ and $\mathbf{c}_{45}$ in $\mathscr{F}_{1}$ can be expressed as:

$$
\begin{aligned}
& { }^{1} \mathbf{v}_{c 35}={ }^{1} \mathbf{v}_{H 5}+\left({ }^{1} \mathbf{h}_{5}-{ }^{1} \mathbf{c}_{35}\right) \times{ }^{1} \omega_{5 / 1} \\
& { }^{1} \mathbf{v}_{c 45}={ }^{1} \mathbf{v}_{H 5}+\left({ }^{1} \mathbf{h}_{5}-{ }^{1} \mathbf{c}_{45}\right) \times{ }^{1} \omega_{5 / 1}
\end{aligned}
$$

From Eqs. (2)-(5) and Eqs. (6)-(7):

$$
\begin{aligned}
& -\dot{\theta}_{3} r_{3}{ }^{1} \mathbf{y}_{2}=-r_{3} \dot{\alpha}^{1} \mathbf{y}_{2}+r_{5}{ }^{1} \mathbf{z}_{2} \times\left(\dot{\alpha}^{1} \mathbf{z}_{2}-\dot{\beta}^{1} \mathbf{x}_{2}\right) \\
& -\dot{\theta}_{4} r_{4}{ }^{1} \mathbf{y}_{2}=-r_{4} \dot{\alpha}^{1} \mathbf{y}_{2}-r_{5}{ }^{1} \mathbf{z}_{2} \times\left(\dot{\alpha}^{1} \mathbf{z}_{2}-\dot{\beta}^{1} \mathbf{x}_{2}\right)
\end{aligned}
$$

Upon simplification of Eqs. (8) and (9), we obtain:

$$
\begin{aligned}
& \dot{\theta}_{3}=\dot{\alpha}+\mu \dot{\beta} \\
& \dot{\theta}_{4}=\dot{\alpha}-\mu \dot{\beta}
\end{aligned}
$$




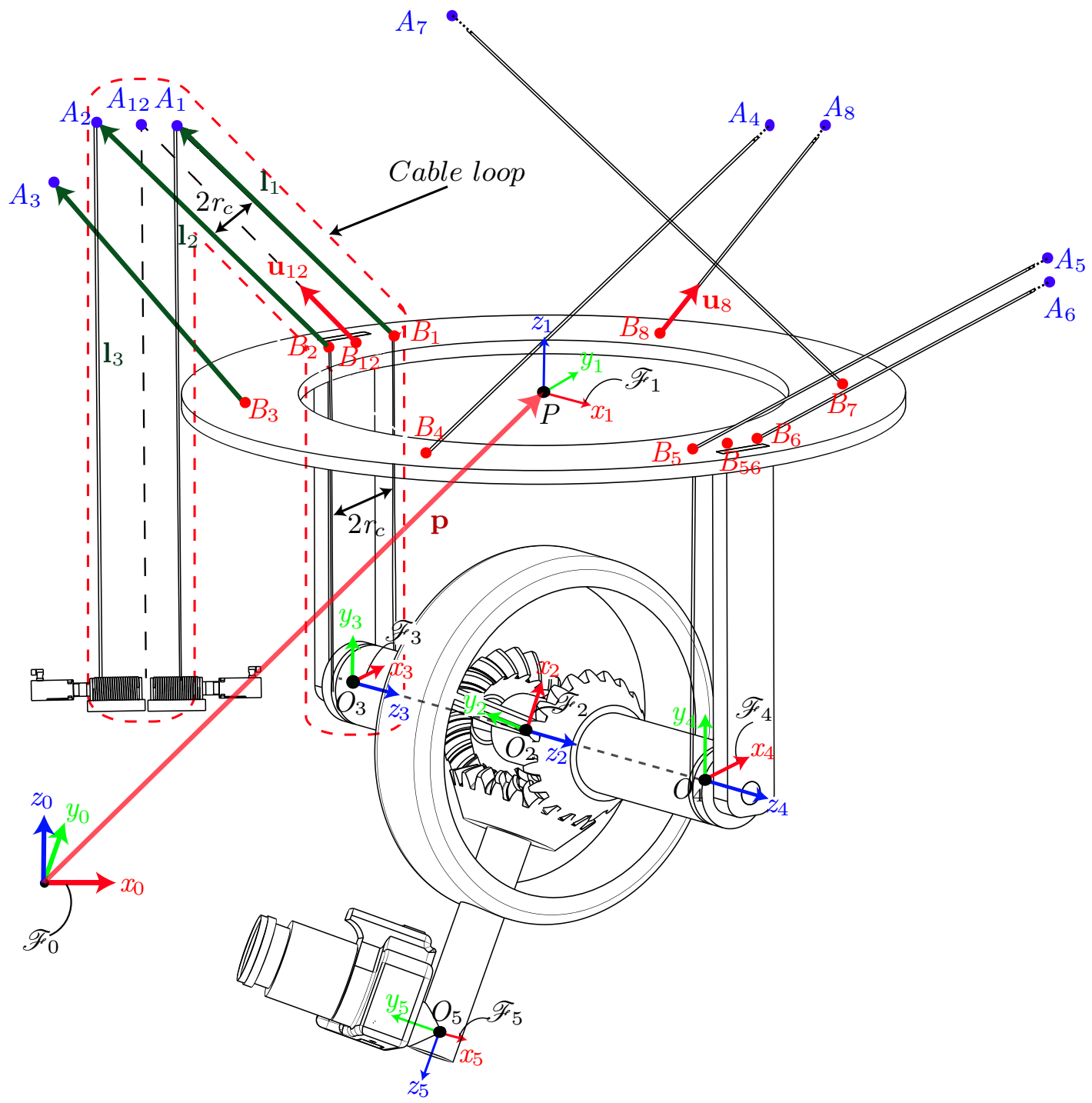

Fig. 3: Schematic of the moving-platform with an embedded camera
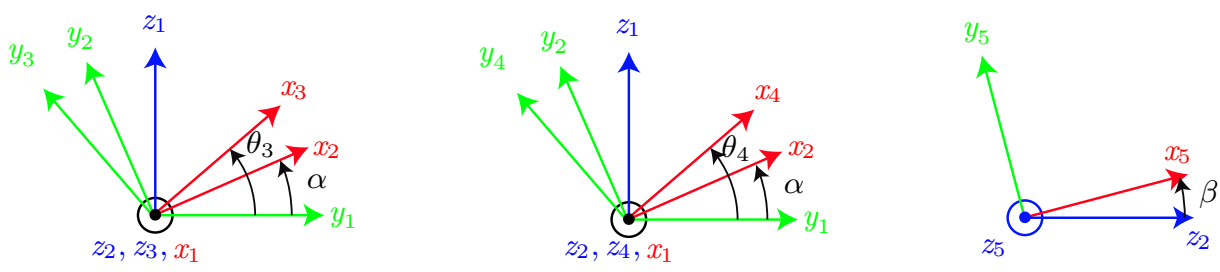

Fig. 4: Angles $\alpha, \beta, \theta_{3}$ and $\theta_{4}$ 
As a result, the output rotational velocity vector $\dot{\mathbf{q}}_{T R}$ of the tilt-roll wrist is expressed as a function of its input velocity vector $\dot{\boldsymbol{\theta}}$ in a matrix form as follows:

$$
\dot{\mathbf{q}}_{T R}=\mathbf{J}_{T R} \dot{\boldsymbol{\theta}}
$$

with $\mathbf{J}_{T R}$ is the kinematic Jacobian matrix of the wrist expressed as:

$$
\mathbf{J}_{T R}=\left[\begin{array}{cc}
1 / 2 & 1 / 2 \\
1 / 2 \mu & -1 / 2 \mu
\end{array}\right]
$$

$\dot{\mathbf{q}}_{T R}=[\dot{\alpha}, \dot{\beta}]^{T}$ and $\dot{\boldsymbol{\theta}}=\left[\dot{\theta}_{3}, \dot{\theta}_{4}\right]^{T}$.

Let $\mathbf{m}=\left[m_{\alpha}, m_{\beta}\right]^{T}$ be the output moments of the tilt-roll wrist and $\boldsymbol{\tau}=$ $\left[\tau_{3}, \tau_{4}\right]^{T}$ be the input torque vector of the wrist. From Eq. (12),

$$
\boldsymbol{\tau}=-\mathbf{J}_{T R}^{T} \mathbf{m}_{e}
$$

with $\mathbf{m}_{e}=-\mathbf{m}$ being the external moments applied by environment to the tiltroll wrist. By rewriting Eq. (14) we can express the static equilibrium of the tilt-roll wrist as follows:

$$
\mathbf{m}_{e}=\mathbf{W}_{T R} \boldsymbol{\tau}
$$

with $\mathbf{W}_{T R}$ being the wrench matrix of the wrist:

$$
\mathbf{W}_{T R}=-\mathbf{J}_{T R}^{-T}
$$

Besides $\mathbf{m}_{e}$ is expressed as:

$$
\mathbf{m}_{e}=\left[\begin{array}{lll}
m_{2} g h_{2} \cos \alpha & m_{2} g w_{5} \cos \alpha \sin \beta
\end{array}\right]^{T}
$$

$m_{2}$ indicates the mass of $\mathscr{P}_{5}$ and $\mathbf{g}=[0,0,-g]^{T}$ is the gravity acceleration with $g=9.81 \mathrm{~m} . \mathrm{s}^{-2}$. As shown in Fig. $2, h_{2}$ and $w_{5}$ denotes the distances between $\mathrm{O}_{2}$ and $C_{2}$ along $z_{5}$ and $y_{5}$, respectively. $C_{2}$ is the Center of Mass (CoM) of the end-effector.

\section{Kinetostatic Model of the Manipulator}

In this section we present the kinetostatic model of the overall manipulator. In order to define the manipulator Jacobian and its wrench matrix, we first introduce the loop-closure equations of the CDPR, which are given by

$$
{ }^{0} \mathbf{l}_{i}={ }^{0} \mathbf{a}_{i}-{ }^{0} \mathbf{p}-{ }^{0} \mathbf{R}_{1}{ }^{1} \mathbf{b}_{i}, \quad i=1,2, \ldots, 8
$$

where ${ }^{0} \mathbf{l}_{i}$ is the $i$ th cable vector, i.e., the Cartesian coordinate vector pointing from point $B_{i}$ to point $A_{i}$. Points $A_{i}$ and $B_{i}$ stand for the $i$ th cable exit point and anchor point, respectively. The former point is the location of the $i$ th pulley fixed to the ceiling and the latter is the connection point between the cable and the MP. ${ }^{0} \mathbf{a}_{i}=\left[\begin{array}{lll}a_{i x} & a_{i y}, & a_{i z}\end{array}\right]^{T},{ }^{1} \mathbf{b}_{i}=\left[\begin{array}{lll}b_{i x} & b_{i y}, & b_{i z}\end{array}\right]^{T}$ and ${ }^{0} \mathbf{p}=\left[\begin{array}{ll}p_{x} & p_{y}, p_{z}\end{array}\right]^{T}$ are the Cartesian coordinate vector of points $A_{i}, B_{i}$ and $P$, respectively. $\mathbf{t}_{i}$, 
$i=1,2, \ldots, 8$, stands for the $i$ th cable tension vector. $\mathbf{t}_{i}=t_{i}{ }^{0} \mathbf{u}_{i}$ and its magnitude is expressed as $t_{i}=\left\|\mathbf{t}_{i}\right\|_{2}, i=1, \ldots, 8 .{ }^{0} \mathbf{u}_{i}$ denotes the $i$ th cable unit vector namely,

$$
{ }^{0} \mathbf{u}_{i}=\frac{{ }^{0} \mathbf{l}_{i}}{l_{i}}, \quad i=1,2, \ldots, 8
$$

$l_{i}$ being the $i$ th cable length.

In the modeling, one simple virtual cable substitutes each cable loop. The latter virtual cables replace each cable loop with $A_{i}$ and $B_{i}, i=12,56$, as their exit point and anchor points, respectively. As long as the MP is far from points $A_{1}$ and $A_{2}$, then $A_{1} B_{1}$ and $A_{2} B_{2}$ can be assumed parallel and the virtual cable model replaces the first cable loop. This analogy also holds for second cable loop. Therefore, the effect of first cable loop onto the MP is the force passing through the midpoint $B_{12}$ between $B_{1}$ and $B_{2}$ along the unit vector $\mathbf{u}_{12}$ of segment $A_{12} B_{12} \cdot \mathbf{t}_{12}=\mathbf{t}_{1}+\mathbf{t}_{2}$ is the tension resultant in the first cable loop. The Cartesian coordinates of the exit and anchor points in both the cable loops are:

$$
\begin{aligned}
& \mathbf{a}_{12}=\left(\mathbf{a}_{1}+\mathbf{a}_{2}\right) / 2 \\
& \mathbf{a}_{56}=\left(\mathbf{a}_{5}+\mathbf{a}_{6}\right) / 2
\end{aligned}
$$

and,

$$
\begin{aligned}
& \mathbf{b}_{12}=\left(\mathbf{b}_{1}+\mathbf{b}_{2}\right) / 2 \\
& \mathbf{b}_{56}=\left(\mathbf{b}_{5}+\mathbf{b}_{6}\right) / 2
\end{aligned}
$$

equilibrium of the external forces applied on the MP, is formulated as follows:

$$
\sum t_{i}{ }^{0} \mathbf{u}_{i}+m \mathbf{g}=0, \quad i=12,3,4,56,7,8
$$

$m \mathbf{g}$ is the weight of the MP. $m$ denotes total mass of the MP and the spherical wrist. The equilibrium of moments applied onto the MP about point $P$ expressed in frame $\mathscr{F}_{0}$ takes the form:

$$
\sum t_{i}\left({ }^{0} \mathbf{R}_{1}{ }^{1} \mathbf{b}_{i} \times{ }^{0} \mathbf{u}_{i}\right)+m\left({ }^{0} \mathbf{c}-{ }^{0} \mathbf{p}\right) \times{ }^{0} \mathbf{g}=0, \quad i=1, \ldots, 8
$$

with $\mathbf{c}$ being the Cartesian coordinates vector of the CoM of MP:

$$
\mathbf{c}=\frac{m_{1} \mathbf{c}_{1}+m_{2} \mathbf{c}_{2}}{m_{1}+m_{2}}
$$

$\mathbf{c}_{1}$ stands for the CoM of components $\mathscr{P}_{1}$ to $\mathscr{P}_{4}$ of mass of $m_{1}$ and $\mathbf{c}_{2}$ denotes the CoM of $\mathscr{P}_{5}$ of mass of $m_{2}$. Therefore, overall mass of the MP is expressed as follows:

$$
m=m_{1}+m_{2}
$$

The input tilt-roll wrist torques $\tau_{3}$ and $\tau_{4}$ are a function of the cable tension difference in cable loops $\mathscr{C}_{12}$ and $\mathscr{C}_{56}$, respectively:

$$
\begin{aligned}
& \tau_{3}=r_{c}\left(t_{1}-t_{2}\right) \\
& \tau_{4}=r_{c}\left(t_{6}-t_{5}\right)
\end{aligned}
$$


$r_{c}$ being the radius of the groove made in $\mathscr{P}_{3}$ and $\mathscr{P}_{4}$ to house the two cable loops. From Eq. (15) and Eqs. (24) to (29) the static equilibrium model of the manipulator is expressed in a matrix form as:

$$
\mathbf{W t}+\mathbf{w}_{g}=\mathbf{0}_{8}
$$

where $\mathbf{0}_{8}$ is a eight-dimensional zero vector and the wrench matrix $\mathbf{W}$ takes the following form:

$$
\mathbf{W}=\left[\begin{array}{cccccccc}
{ }^{0} \mathbf{u}_{12} & { }^{0} \mathbf{u}_{12} & { }^{0} \mathbf{u}_{3} & { }^{0} \mathbf{u}_{4} & { }^{0} \mathbf{u}_{56} & { }^{0} \mathbf{u}_{56} & { }^{0} \mathbf{u}_{7} & { }^{0} \mathbf{u}_{8} \\
{ }^{0} \mathbf{d}_{1} & { }^{0} \mathbf{d}_{2} & { }^{0} \mathbf{d}_{3}{ }^{0} \mathbf{d}_{4} & { }^{0} \mathbf{d}_{5} & { }^{0} \mathbf{d}_{6} & { }^{0} \mathbf{d}_{7} & { }^{0} \mathbf{d}_{8} \\
r_{c} & -r_{c} & 0 & 0 & -r_{c} & r_{c} & 0 & 0 \\
\mu r_{c} & -\mu r_{c} & 0 & 0 & \mu r_{c} & -\mu r_{c} & 0 & 0
\end{array}\right]
$$

with,

$$
{ }^{0} \mathbf{d}_{i}={ }^{0} \mathbf{R}_{1}{ }^{1} \mathbf{b}_{i} \times{ }^{0} \mathbf{u}_{i}, \quad i=1, \ldots, 8
$$

$\mathbf{t}$ is the vector containing the tensions exerted by the eight actuators to the cables.

$$
\mathbf{t}=\left[\begin{array}{llllllll}
t_{1} & t_{2} & t_{3} & t_{4} & t_{5} & t_{6} & t_{7} & t_{8}
\end{array}\right]^{T}
$$

$\mathbf{w}_{g}$ is the eight-dimension gravity wrench vector applied on the MP and tilt-roll wrist, namely,

$$
\mathbf{w}_{g}=\left[m^{0} \mathbf{g}^{T} \quad m\left({ }^{0} \mathbf{R}_{1}{ }^{1} \mathbf{c} \times{ }^{0} \mathbf{g}\right)^{T} \mathbf{m}_{e}^{T}\right]^{T}
$$

It should be noted that, in Eq. (30) the friction between cable-loops and $\mathscr{P}_{3}$ and $\mathscr{P}_{4}$ is neglected. Hereafter, we can express the differential kinematics of the manipulator that formulates the relation between the output twist $\dot{\mathbf{q}}=$ $\left[\dot{\mathbf{p}}^{T}, \boldsymbol{\omega}^{T}, \dot{\mathbf{q}}_{T R}^{T}\right]^{T}$ of the manipulator and the cable vector $\mathbf{i}=\left[i_{1}, \ldots, \dot{l}_{8}\right]^{T}$ :

$$
\mathbf{J} \dot{\mathbf{q}}=\mathbf{i}
$$

Velocity of the origin of frame $\mathscr{F}_{1}$ with respect to $\mathscr{F}_{0}$ is defined as $\dot{\mathbf{p}}$ and the angular velocity of the MP with respect to $\mathscr{F}_{0}$ is defined as $\boldsymbol{\omega}$. Jacobian matrix $\mathbf{J}$ is calculated based on the well-known kineto-static duality:

$$
\mathbf{J}=-\mathbf{W}^{T}
$$

\section{$5 \quad$ Workspace Analysis}

The section deals with the workspace analysis of the CDPR using a tilt-roll wrist under study.

The set $\mathscr{T}$ forms the feasible cable tensions as a box in eight-dimensional space:

$$
\mathscr{T}=\left\{\mathbf{t} \in \mathbb{R}^{8}: \mathbf{t}_{\min } \leq \mathbf{t} \leq \mathbf{t}_{\max }\right\} .
$$

where, $\mathbf{t}_{\min }$ and $\mathbf{t}_{\max }$ are the lower and upper bounds of admissible cable tensions. Static Workspace (SW), namely, $\mathscr{S}$ is set of MP poses and tilt-roll wrist 
configurations satisfying the static equilibrium of the manipulator with admissible cable tensions.

$$
\mathscr{S}=\left\{\left({ }^{0} \mathbf{p},{ }^{0} \mathbf{R}_{1}, \mathbf{q}_{T R}\right) \in \mathbb{R}^{3} \times S O(3) \times \in \mathbb{R}^{2}: \exists \mathbf{t} \in \mathscr{T}, \mathbf{W t}+\mathbf{w}_{g}=\mathbf{0}_{8}\right\}
$$

where $S O(3)$ is the group of proper rotation matrices. From Eq. (38) we can derive two subsets $\mathscr{S}_{1}$ and $\mathscr{S}_{2}$ based on the constrained orientations of the MP and the tilt-roll wrist. The former subset, namely, $\mathscr{S}_{1}$ is a set for a given orientation of the MP and the wrist, i.e., the translational SW of the manipulator with constant orientation of ${ }^{0} \mathbf{R}_{1}$ and $\mathbf{q}_{T R}$.

$$
\mathscr{S}_{1}=\left\{{ }^{0} \mathbf{p} \in \mathbb{R}^{3} \mid\left({ }^{0} \mathbf{R}_{1}, \alpha, \beta\right) \text { given }: \exists \mathbf{t} \in \mathscr{T}, \mathbf{W t}+\mathbf{w}_{g}=\mathbf{0}_{8}\right\}
$$

$\mathscr{S}_{2}$ amounts to the SW of the manipulator for a constant orientation of the MP while the tilt and roll is free to rotate.

$$
\mathscr{S}_{2}=\left\{\left.{ }^{0} \mathbf{p} \in \mathbb{R}^{3}\right|^{0} \mathbf{R}_{1} \text { given }:-\pi \leq \alpha \& \beta \leq \pi: \exists \mathbf{t} \in \mathscr{T}, \mathbf{W t}+\mathbf{w}_{g}=\mathbf{0}_{8}\right\}
$$

Here, we consider CREATOR prototype introduced in [10] in order to trace $\mathscr{S}_{1}$ and $\mathscr{S}_{2}$ for different orientations of the moving-platform. The main dimensions of the prototype are given in Table 1. The specifications listed in Table 1 are based on the preliminarily manufactured prototype shown in Fig. 6. The weight of the wrist highly depends on the material of the parts $\mathscr{P}_{2}, \mathscr{P}_{5}$ and the end-effector. Because ABS plastic is employed for $\mathscr{P}_{2}$, the overall weight of the MP can be significantly reduced by substituting steel bevel gears with nylon ones. The prototype frame is $4 \mathrm{~m}$ long $\left(l_{0}\right), 3.5 \mathrm{~m}$ wide $\left(w_{0}\right)$ and $4 \mathrm{~m}$ high $\left(h_{0}\right)$. The MP under study is suspended by six cables as shown in Fig. 1. The SW

Table 1: Parameters of CREATOR prototype

\begin{tabular}{lcl}
\hline \hline Parameter & Abbreviation & Value \\
\hline Vector of CoM associated to $\mathscr{P}_{1-\mathscr{P}_{4}[\mathrm{~m}]}{ }^{1} \mathbf{c}_{1}$ & {$[0,0,-0.15]^{T}$} \\
Vector of CoM associated to $\mathscr{P}_{5}[\mathrm{~m}]$ & ${ }^{2} \mathbf{c}_{2}$ & {$\left[-h_{2}, w_{5} \cos \beta,-w_{5} \sin \beta\right]^{T}$} \\
Mass of $\mathscr{P}_{1-\mathscr{P}_{4}[\mathrm{~kg}]}$ & $m_{1}$ & 4 \\
Mass of $\mathscr{P}_{5}[\mathrm{~kg}]$ & $m_{2}$ & 0.6 \\
Radius of the groove made in $\mathscr{P}_{3}$ and $\mathscr{P}_{4}[\mathrm{~m}]$ & $r_{c}$ & 0.1 \\
Distance between $\mathrm{O}_{2}$ and $C_{2}$ along $z_{5}[\mathrm{~m}]$ & $h_{2}$ & 0.23 \\
Distance between $\mathrm{O}_{2}$ and $C_{2}$ along $y_{5}[\mathrm{~m}]$ & $w_{5}$ & 0.03 \\
Maximum admissible cable tension $[\mathrm{N}]$ & $t_{\max }$ & 128 \\
Minimum admissible cable tension $[\mathrm{N}]$ & $t_{\min }$ & 0 \\
\hline \hline
\end{tabular}

illustrated in Fig. 5 refers to constant orientation of the MP for different given orientation of MP. Cable loops are plotted in magenta and single-actuated cables are represented in red. It is apparent that $\psi$ has significant effect on the size of the SW as well as the position of anchor points and exit points. According to the obtained results, the largest SW (fig. 5b) is associated with $\psi=0^{\circ}$. The gravitational external wrench due to the varying CoM of the wrist, namely $C_{2}$ has effect 
$\mathscr{S}_{1}$ with $\alpha=\pi / 2$ and $\beta=0$ (home configuration of the wrist)

$\mathscr{S}_{2}$ with $-\pi \leq \alpha \leq \pi,-\pi \leq \beta \leq \pi$
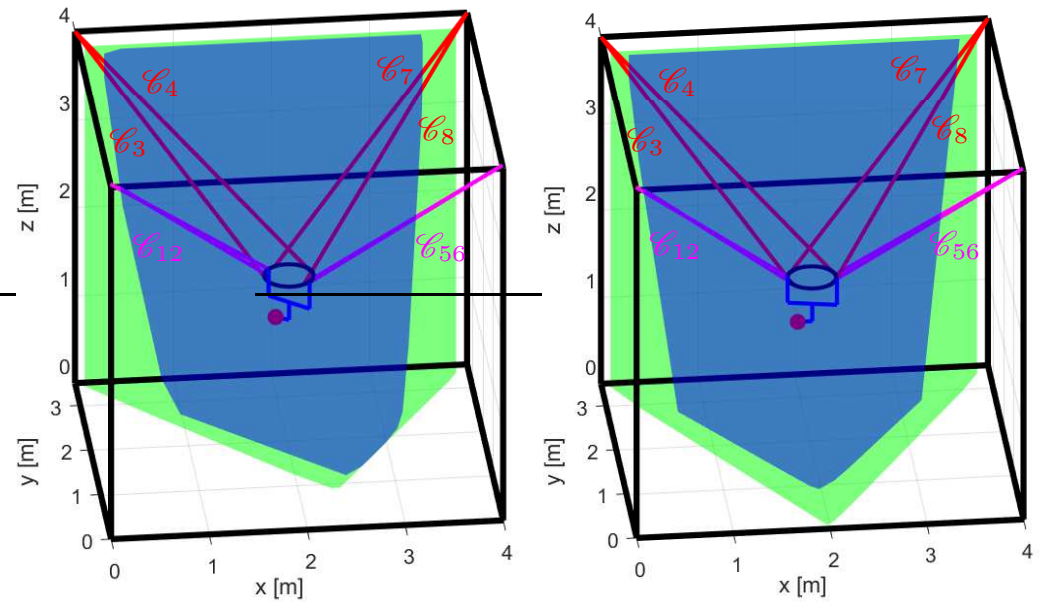

(a) SW with $\phi=0^{\circ}, \chi=0^{\circ}$ and $\psi=-30^{\circ}$

(b) SW with $\phi=0^{\circ}, \chi=0^{\circ}$ and $\psi=0^{\circ}$
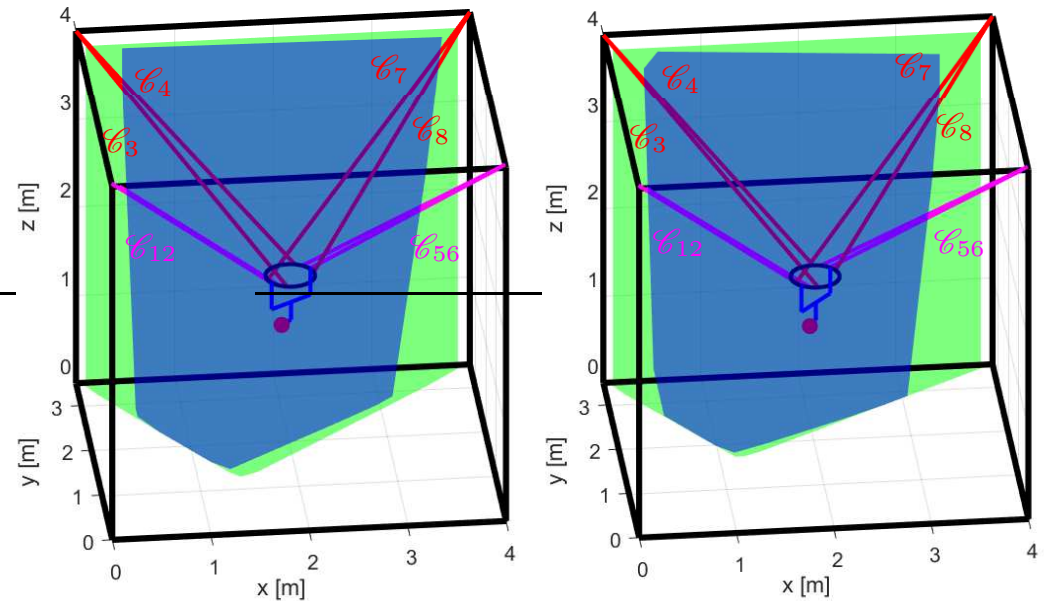

(c) SW with $\phi=0^{\circ}, \chi=0^{\circ}$ and $\psi=45^{\circ}$

(d) SW with $\phi=0^{\circ}, \chi=0^{\circ}$ and $\psi=60^{\circ}$
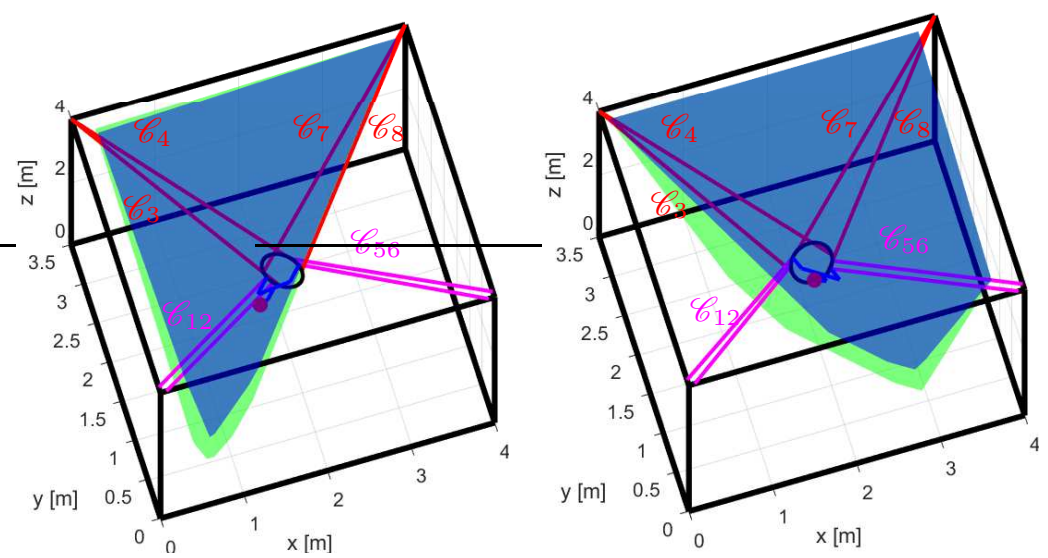

(e) SW with $\phi=0^{\circ}, \chi=30^{\circ}$ and $\psi=30^{\circ}$ (f) SW with $\phi=0^{\circ}, \chi=-20^{\circ}$ and $\psi=$

Fig. 5: $\mathscr{S}_{1}$ and $\mathscr{S}_{2}$ for different orientations of the moving-platform 
on the SW. Therefore, two cases are considered. The former case (shown with green volume in the figure) considers the minimum external wrench applied onto the MP by the wrist, i.e., the tilt-roll wrist is in home configuration $(\alpha=\pi / 2$ and $\beta=0$ ). The latter case computes the $\mathrm{SW}$ while the $\mathscr{P}_{5}$ exerts the maximum external wrench due to gravity on the MP and the wrist with blue volume in the figure. It should be noted that, the gravitational external wrench of the wrist is a function of $\alpha$ and $\beta$. The figure shows the variation of $\mathscr{S}_{1}$ and $\mathscr{S}_{2}$ for different orientation of MP. The largest volumes for $\mathscr{S}_{1}$ and $\mathscr{S}_{2}$ are $25.3 \mathrm{~m}^{3}$ and $14.15 \mathrm{~m}^{3}$, respectively, as shown in Fig. 5(b). On the other hand, the smallest volume of SW is associated to Fig. 5(d) with $13 \mathrm{~m}^{3}$ of $\mathscr{S}_{1}$ and $8.15 \mathrm{~m}^{3}$ of $\mathscr{S}_{2}$.

It appears to be a trade-off between translation and large rotation workspaces due to the cable-loops in the fully-actuated CDPR. It is noteworthy that translation and orientation workspaces become larger when employing two additional actuators.

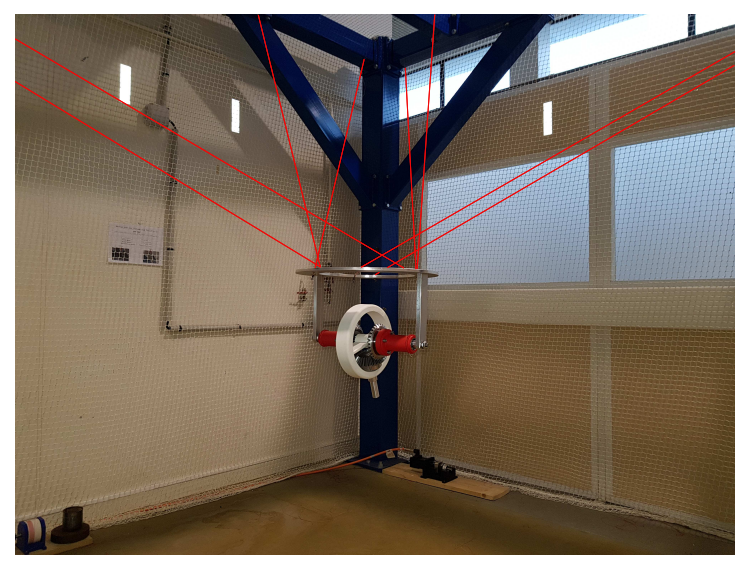

Fig. 6: Prototype of the CDPR with an embedded tilt-roll wrist

\section{Conclusions}

A Cable-Driven Parallel Robot (CDPR) using a Tilt-Roll (T\&R) wrist has been introduced in this paper. The robot combines the advantages of CDPR in terms of large translational workspace with those of tilt-roll wrist in terms of large rotational amplitudes. The robot uses cables to transmit power directly from fixed motors on the ground to the tilt-roll wrist. In comparison with conventional cable-driven parallel robots and cable-driven suspended robots, the proposed concept adds singularity-free and large tilt-roll motions to the end-effector. This leads to a trade-off, however, between translational and rotational workspaces due to tension coupling in cable-loops for fully-actuated CDPRs. The kinetostatic model of the proposed manipulator was studied. The static workspace of 
the manipulator was also defined and analyzed. The detailed design, prototyping and dynamic modeling of the cable-driven parallel robot equipped with a tilt-roll wrist are left for future work. Moreover, the study of the over-actuated CDPRs with the embedded tilt-roll wrist will be carried out in order to maximize their workspaces.

\section{References}

1. S. Bai and J. Angeles. The design of a gearless pitch-roll wrist. In Robotics and Automation, 2005. ICRA 2005. Proceedings of the 2005 IEEE International Conference on, pages 3213-3218. IEEE, 2005.

2. F. Guay, P. Cardou, A. L. Cruz-Ruiz, and S. Caro. Measuring how well a structure supports varying external wrenches. In New Advances in Mechanisms, Transmissions and Applications, pages 385-392. Springer, 2014.

3. K. Hyodo and H. Kobayashi. Kinematic and control issues on tendon controlled wrist mechanism. In Robotics, Mechatronics and Manufacturing Systems edited by T. Takamori, K. Tsuchiya, pages 89-94. Elsevier, 1992.

4. H. Khakpour and L. Birglen. Workspace augmentation of spatial 3-dof cable parallel robots using differential actuation. In Intelligent Robots and Systems (IROS 2014), 2014 IEEE/RSJ International Conference on, pages 3880-3885. IEEE, 2014.

5. H. Khakpour, L. Birglen, and S.-A. Tahan. Synthesis of differentially driven planar cable parallel manipulators. IEEE Transactions on Robotics, 30(3):619-630, 2014.

6. H. Khakpour, L. Birglen, and S.-A. Tahan. Analysis and optimization of a new differentially driven cable parallel robot. Journal of Mechanisms and Robotics, $7(3): 034503,2015$.

7. T. N. Le, H. Dobashi, and K. Nagai. Configuration of redundant drive wire mechanism using double actuator modules. ROBOMECH Journal, 3(1):25, 2016.

8. T. N. Le, H. Dobashi, and K. Nagai. Kinematical and static force analysis on redundant drive wire mechanism with velocity constraint modules to reduce the number of actuators. ROBOMECH Journal, 3(1):22, 2016.

9. S. Lessanibahri, P. Cardou, and S. Caro. Parasitic inclinations in cable-driven parallel robots using cable loops. Procedia CIRP, 70:296-301, 2017.

10. S. Lessanibahri, P. Cardou, and S. Caro. Kinetostatic analysis of a simple cabledriven parallel crane. In Proceedings of the ASME 2018 International Design Engineering Technical Conferences $\&$ Computers and Information in Engineering Conference IDETC/CIE 2018, Quebec city, Canada, August 28, 2018.

11. K. Nagai, T. N. Le, Y. Hayashi, and K. Ito. Kinematical analysis of redundant drive wire mechanisms with velocity constraint. In Mechatronics and Automation (ICMA), 2012 International Conference on, pages 1496-1501. IEEE, 2012.

12. A. Platis, T. Rasheed, P. Cardou, and S. Caro. Isotropic design of the spherical wrist of a cable-driven parallel robot. In Advances in Robot Kinematics 2016, pages 321-330. Springer, 2018.

13. P. Racioppo, W. Saab, and P. Ben-Tzvi. Design and analysis of reduced degree of freedom modular snake robot. In ASME 2017 International Design Engineering Technical Conferences and Computers and Information in Engineering Conference, pages V05BT08A009-V05BT08A009. American Society of Mechanical Engineers, 2017.

14. A. L. C. Ruiz, S. Caro, P. Cardou, and F. Guay. Arachnis: Analysis of robots actuated by cables with handy and neat interface software. In Cable-Driven Parallel Robots, pages 293-305. Springer, 2015. 